\title{
A Deployable Mechanism Concept for the Collection of Small-to-Medium-Size Space Debris
}

\author{
David St-Onge ${ }^{\mathrm{a}, *}$, Inna Sharf ${ }^{\mathrm{b}}$, Luc Sagnières ${ }^{\mathrm{b}}$, Clément Gosselin ${ }^{\mathrm{a}}$ \\ ${ }^{a}$ Department of Mechanical Engineering, Laval University, 1065 Médecine Av., Québec \\ G1V 0A6, Qc, Canada \\ ${ }^{b}$ Department of Mechanical Engineering, McGill University, 3480 University St., Montréal \\ H3A 0E9, Qc, Canada
}

\begin{abstract}
Current efforts in active debris removal strategies and mission planning focus on removing the largest, most massive debris. It can be argued, however, that small untrackable debris, specifically those smaller than $5 \mathrm{~cm}$ in size, also pose a serious threat. In this work, we propose and analyze a mission to sweep the most crowded Low Earth Orbit with a large cupola device to remove small-tomedium-size debris. The cupola consists of a deployable mechanism expanding more than 25 times its storage size to extend a membrane covering its surface. The membrane is sufficiently stiff to capture most small debris and to slow down the medium-size objects, thus accelerating their fall. An overview of the design of a belt-driven rigid-link mechanism proposed to support the collecting cupola surface is presented, based on our previous work. Because of its large size, the cupola will be subject to significant aerodynamic drag; thus, orbit maintenance analysis is carried out using the DTM-2013 atmospheric density model and it predicts feasible requirements. While in operation, the device will also be subject to numerous hyper-velocity impacts which may significantly perturb its orientation from the desired attitude for debris collection. Thus, another important feature of the proposed debris removal device is a distributed array of flywheels mounted on the cupola for reorienting and stabilizing its attitude during the mission. Analysis using a stochastic modeling framework for hyper-velocity impacts demonstrates that three-axes attitude stabilization is achievable with the flywheels array. MASTER-2009 software is employed to provide relevant data for all debris related estimates, including the debris fluxes for the baseline mission design and for assessment of its expected performance. Space debris removal is a high priority for ensuring sustainability of space and continual launch and operation of man-made space assets. This manuscript presents the first analysis of a small-to-medium size debris removal mission, albeit finding it to not be economically viable at the present time.
\end{abstract}

Keywords: space debris, deployable mechanism, active debris removal, small size, cupola device

PACS: 91.10.Sp, 95.40.+s 


\section{Introduction}

Development and launch of a large scientific, communications, or military satellite can cost hundreds of millions of dollars for design, construction, and once launched, monitoring and operation. The cost estimate for the recently launched GOES-R geostationary weather satellite is a billion dollars. These large investments are already exposed to high technological and management risks, and are now subject to another potential threat: the growing orbital debris population.

The most favored orbits, below $2000 \mathrm{~km}$ altitude from the Earth's surface, are also the most crowded with debris. Their proximity makes them more affordable and allows for low-latency communications with ground-based infrastructure. These LEO (Low Earth Orbits) are mainly used for Earth observation satellites, telecommunication networks and manned missions (Krag et al. (2007)). Unfortunately, a multitude and wide variety of debris objects populate these orbits: rocket stages from old space missions, inoperable satellites, and all sizes of particles, down to $O\left(10^{-15}\right) \mathrm{kg}$ mass, generated by natural (mainly, meteoroid) and man-made sources. There is strong consensus (Vance and Mense (2013), Liou (2011a)) that particles as small as $0.5 \mathrm{~cm}$ in size are potentially lethal to the operating and newly launched spacecraft; if disabled by such a lethal impact, these craft would contribute to the growth of large debris orbital population. With this perspective, we develop and analyze a solution to remove untrackable small-to-medium size debris objects - those less than $5 \mathrm{~cm}$-which, combined with the current debris mitigation measures and with the anticipated large debris removal efforts, will contribute to the safety of future space missions.

In Section 2 of this paper, we present the state of the debris population in lower Earth orbits with a particular focus on the small-to-medium size debris situation. The current technologies proposed by research groups around the world for Active Debris Removal (ADR) are then reviewed to reveal the scarcity of solutions for this category of debris. Then, an Active Small-to-Medium-Size Debris Removal (ASMDR) solution based on a cupola assembly of belt-driven triangular deployable mechanisms, originally presented in St-Onge and Gosselin (2016b), is proposed and tailored to fit the small-to-medium debris removal mission. The unique size of the proposed device greatly influences both its orbital and attitude dynamics. Thus, predictions of aerodynamic drag expected at the device altitude are presented and estimates for orbital maintenance in terms of $\Delta v$ budget are computed. Due to the symmetrical configuration of the device, we expect the predominant attitude perturbation to result from impacts with medium-sized debris, as they are encountered. To negate these effects, so that the device maintains its optimal orientation, the distributed flywheel architecture presented in St-Onge and Gosselin (2016a) is adapted for

\footnotetext{
${ }^{*}$ Corresponding author

Email addresses: david.st-onge.2@ulaval.ca (David St-Onge), inna.sharf@mcgill.ca (Inna Sharf), luc.sagnieres@mail.mcgill.ca (Luc Sagnières), gosselin@gmc.ulaval.ca (Clément Gosselin)
} 
this scenario. Stochastic modeling of hyper-velocity impacts is used to predict the expected angular momentum transfer to the device and the results show feasibility of three-axis attitude stabilization with the distributed flywheel array. Finally, the expected performance of the mission in terms of the numbers of debris collected and the costs per collected debris are given and compared to the published value analysis for debris removal (Vance and Mense (2013)).

\section{Small-to-Medium-Sized Orbital Debris}

Based on Kessler's model (Kessler and Cour-Palais (1978)), the orbital debris population is likely to grow at an increasing rate over the next 200 years, as any one collision generates a multitude of new debris of smaller size. This phenomenon was observed in the widely quoted 2009 incident where a commercial satellite, Iridium-33 $(560 \mathrm{~kg})$, hit a defunct Russian military satellite, Cosmos-2251 (900 kg) (Bonnal et al. (2013)). Their collision created close to 2,000 pieces of trackable $(>10 \mathrm{~cm})$ new debris. The number of secondary debris, as predicted by the NASA breakup model of this collision, is 3.76 million pieces larger than $1 \mathrm{~mm}$ in size, of which 73,320 are over $1 \mathrm{~cm}$ (Ting Wang (2010)). A significant number of debris is also generated from the explosions or breakup events of defunct spacecraft. One such occurrence in late 2015 is the breakup of the NOAA-16 decommissioned weather satellite, producing 136 catalogued objects (Anz-Meador and Shoots (2016)), and likely tens of thousands of smaller debris.

To deal with the growth of the debris population, several national space agencies are supporting scientific and technological research on ADR systems for retrieval of the largest objects in orbit. The motivation for this is clear since the top $1 \%$ of the largest debris account for $99 \%$ of the total mass of orbital debris (Liou (2011b)). As already noted, a collision with a sizeable object may not only destroy a functional satellite, but it will leave behind a cloud of smaller, but still potentially dangerous objects in orbit. These clouds spread over time and thus greatly increase the probability of a collision with other satellites or debris. While avoidance maneuvers can be planned by satellite operators to avoid a trackable object, this option is not available for mitigating collisions with the numerous untrackable debris.

To estimate the numbers of smaller debris populations, we resort to the state-of-the-art model MASTER-2009, developed by ESA for the purpose of predicting collisional risks and the future near-Earth environment. Using this tool for the reference year 2009, we estimate the debris population of size $<5$ $\mathrm{cm}$ (with lower limit of $1 \mu \mathrm{m}$ ) in LEO to be $7.5 \times 10^{12}$ of which over $1.4 \times 10^{6}$ are larger than $0.5 \mathrm{~cm}$. The small size is deceiving as the damage potential of these objects is magnified by their relative velocities, which average in the range of $10-20 \mathrm{~km} / \mathrm{s}$ in LEO. For collisions occurring at these hyper-velocities, an object as small and light as a paint flake can create substantial damage; numerous such occurrences have been analyzed and documented for the Space Shuttle vehicles (Christiansen et al. (2004)). At these hyper-velocities, an impact creates deformations comparable to an explosion, as was demonstrated in an experiment 
reported in ESA with an impactor of size $1.2 \mathrm{~cm}$. The results of a CNES study cited in Bonnal et al. (2013) showed that the risk to the Spot 5 satellite of mission loss over its lifetime was mostly attributable to debris smaller than 5 $\mathrm{cm}$ in size. In the same reference, it was noted that orbital debris remediation efforts should be extended to smaller debris, and not only limited to large debris objects.

Solutions to protect high-valued assets in space from small debris collisions do exist in the form of material shielding as, for example, on the International Space Station (ISS), which is retrofitted with several shielding configurations to withstand collisions with debris up to $1.4 \mathrm{~cm}$ in size. Indeed, research on the development of new shielding materials and mechanical architectures to increase the overall strength limit is ongoing (Williamsen et al. (2008); Christiansen (2003); Ryan and Christiansen (2011)). Liou (2011b) reported that about 1200 objects larger than $1.4 \mathrm{~cm}$ crossed the orbit of ISS, among which 800 were less than $3 \mathrm{~cm}$. In Liou (2011a), the same author states that for most operational spacecraft, "any impact by debris between $5 \mathrm{~mm}$ and $1 \mathrm{~cm}$ is likely to cause mission-ending damage." This statement has since then been supported by other studies (Nakamura et al. (2015); Bauer et al. (2014)).

We employed MASTER-2009 to conduct a focused investigation of small-tomedium size debris situation with the goal of identifying a mission scenario and potential benefits of the small debris removal mission. MASTER-2009 software was preferred over NASA's ORDEM3 engineering model for its spatial density plotting feature and debris modeling down to the size of $1 \mu \mathrm{m}$. Our targeted mission year is 2020 and therefore, results were computed for this year, assuming no mitigation strategies available in the interim (a.k.a., "Business-as-Usual" scenario in MASTER-2009).

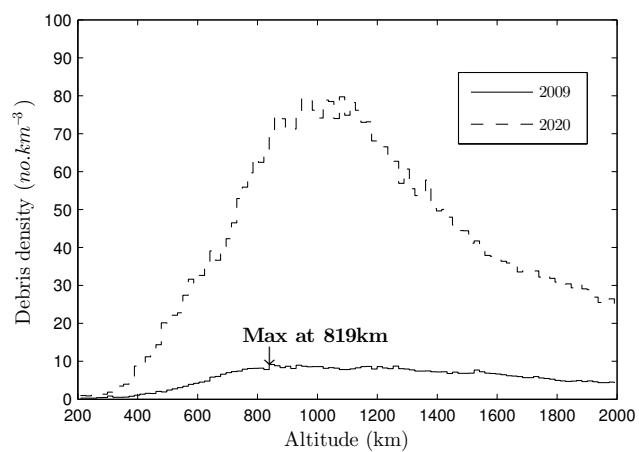

Figure 1: Debris density of objects smaller than $5 \mathrm{~cm}$ in LEO orbits obtained from MASTER2009.

Fig. 1 shows the debris density (excluding meteoroids) for objects smaller than $5 \mathrm{~cm}$ as a function of altitude for LEO, computed in the reference year 2009 and our mission year 2020. Highest population numbers are observed in the $700 \mathrm{~km}$ to $1300 \mathrm{~km}$ altitude band, with the peak 2009 population at $819 \mathrm{~km}$ likely to grow by more than $1000 \%$ by 2020 . Although numerous considerations 
would be weighed in the orbit selection process for a removal mission, such as described in this manuscript, we will use the $819 \mathrm{~km}$ altitude for the mission planning and analysis here. Flegel et al. (2011) indicates that the highest density appears around $900 \mathrm{~km}$ altitude and below this, the small debris naturally decay. We acknowledge that below $800 \mathrm{~km}$ altitudes, debris particles smaller than 1 $\mathrm{mm}$ will experience fast natural orbit decay, as they are strongly affected by the aerodynamic drag (Mandeville et al. (1999)) and that up to $1100 \mathrm{~km}$, dust particles $(<100 \mu m)$ will decay in less than 15 years (Ganguli et al. (2011)). Fig. 2, showing the total debris flux (excluding meteoroids) as a function of size, at $819 \mathrm{~km}$ altitude for year 2020, clearly indicates that most of the debris flux will persist to comprise objects smaller than $5 \mathrm{~cm}$.

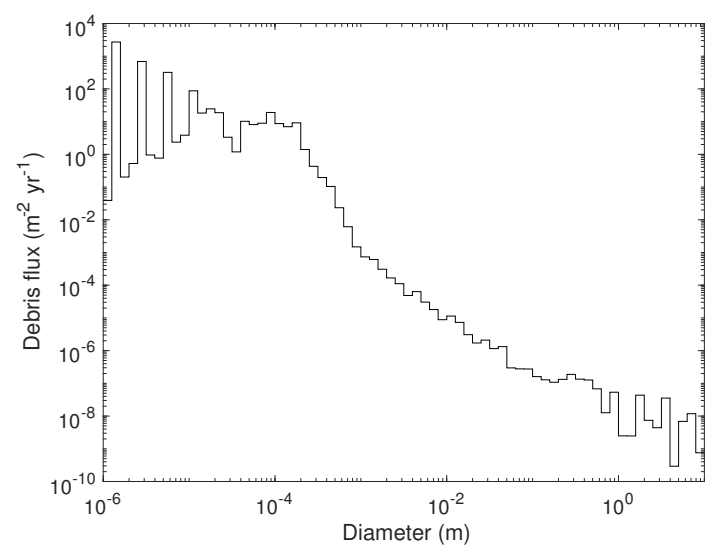

Figure 2: Debris flux predicted for 2020 at $819 \mathrm{~km}$ altitude and $80^{\circ}$ inclination vs. debris diameter obtained from MASTER-2009.

From various orbit simulations in MASTER-2009, it can be surmised that the debris density is higher for orbital inclinations near $\pm 90^{\circ}$, since these orbits all intersect at the Earth's poles, unlike orbits at the other inclinations. Using MASTER-2009 spatial density plotting, one can compute aggregate numbers of objects. Doing this for the entire altitude span of the model from $186 \mathrm{~km}$ to $36786 \mathrm{~km}$, for reference year 2009, one can arrive at the generally accepted number of space debris for every size range ${ }^{1}$. This approach was applied to compute the total number of man-made debris (that is, excluding meteoroids), from $1 \mu \mathrm{m}$ to $5 \mathrm{~cm}$, in the small altitude band $819 \pm 1 \mathrm{~km}$ about the orbit selected for our mission, for years 2009 and 2020. The corresponding results indicate that approximately 84.8 billion small-to-medium-size objects will pose a threat around our nominal orbit in 2020, while in the reference year 2009, the corresponding number is 10.3 billion.

\footnotetext{
${ }^{1}$ http://www.esa.int/Our_Activities/Space_Engineering_Technology/Clean_Space/ How_many_space_debris_objects_are_currently_in_orbit
} 


\section{Active Debris Removal Systems}

Research and development of removal concepts for large debris have been ongoing for over a decade and a number of possible solutions are being considered (Klinkrad and Johnson (2009), Shan et al. (2016), Dudziak et al. (2015)), spanning a full spectrum of technology readiness levels (Barbee et al. (2012)). However, as we argue in the following, none of the concepts proposed for large debris removal are suitable or practical for dealing with small debris.

The most established and technically ready approach for an ADR system, at this time, involves a robotic manipulator mounted on a chaser spacecraft. This technology builds on the knowledge base and flight experience accumulated with robotic manipulators over the course of the Space Shuttle and Space Station programs. Securing a rigid grip on a large tumbling object, such as the defunct satellite, requires accurate prediction of its relative pose and motion to ensure the robot end-effector can approach it safely (Aghili (2012); Nguyen-Huynh and Sharf (2013); Hirzinger et al. (2004)). Relative localization and tracking of debris smaller than $5 \mathrm{~cm}$ in size, to sufficient accuracy, is not likely to be viable in the near future. Furthermore, considering the mission time of retrieving the debris one by one, with a robotic arm, renders this approach impractical for small debris. These observations also apply to Earth based high-power laser concepts proposed to change a large debris' trajectory for it to reach Earth's surface faster (Mason et al. (2011)). This technology used from space could be applied to ablate debris of $1 \mathrm{~cm}$ to $10 \mathrm{~cm}$ in size, as recently proposed in the work of Schmitz et al. (2015), but issues with localizing each single target in orbit still remain.

Several ADR solutions aim to attach a de-orbiting device to the debris, such as an inflatable balloon (Nock et al. (2003)), a sail (Lappas et al. (2011)), or a thrusting module (Barbee et al. (2012)). The issues of localizing the debris to attach a device plague these approaches as well, in addition to the cost of these typically one-target/mission approaches. A highly promising method for debris capture is to use a tethered-net ejected from chaser to envelope the debris (Biesbroek and Innocenti (2013); Zhai and Zhang (2012)) and then to tug it to a disposal orbit. This concept is tailor-made for large tumbling objects, as it does not require close-proximity operations and is robust to net deployment inaccuracies, debris geometry and its rotational motion (Botta et al. (2016)). Another interesting net-based technology involves a net released towards the debris, transferring momentum to it upon contact, thereby modifying its orbit and increasing the aerodynamic drag on it (Taylor et al. (2013)). Applying any one of the net-based concepts to capture a small debris would require an extremely fine meshing of the net, essentially transforming it into a membrane. In the review of Barbee et al. (2012), the use of a "large thin surface material", a.k.a., a membrane, is briefly mentioned as a means of slowing down the debris, but is not pursued further due to the risk of fragmentation.

Finally, a dust shell concept is described in the work of Ganguli et al. (2011) involving the injection of a cloud of micron-size particles into a polar orbit to form a thin dust shell around Earth, to retard small debris through interactions 
with them. At this time, there are many uncertainties associated with this concept, including the spread of the cloud, its orbital and collision mechanics, potential risks to operational assets and the overall effectiveness of the concept. The use of a gaseous cloud created at least $100 \mathrm{~km}$ above Earth to slow down small debris is also the subject of a patent (Dunn (2014)).

\section{ASMDR Proposed Solution}

Motivated by the above considerations and concepts, the technical solution proposed in this paper involves a very large membrane, stretched and supported by a multi-degree-of-freedom mechanism deployed in orbit in a cupola shape, based on the design in the work of St-Onge and Gosselin (2016b), to slow down and capture the debris. The expected result is the removal of approximately $1.2 \%$ of the debris populating the target orbit in a one year mission, using an Ariane 5 rocket to reach the orbit.

\subsection{Mission definition and parameters}

The most important parameters of a "cleaning" mission using a large passive system that would intercept numerous small objects crossing its path are: the area traversed by the device, the time in orbit and the selected orbit. For the baseline mission scenario put forward here, we choose the target orbit to be that most crowded with small to medium sized debris, as discussed previously: the orbit at $819 \mathrm{~km}$ altitude.

The average velocity in this orbit is $7.44 \mathrm{~km} / \mathrm{s}$ and the length of the orbital trajectory is $45,220 \mathrm{~km}$. The device placed in this orbit will perform a full rotation in approximately 1.7 hours. The orbit's longitude of the ascending node does not have much impact on the system performance, but its inclination must be close to $\pm 90^{\circ}$ since we expect higher local debris densities to be over the poles.

The system must be dimensioned so that in its launch configuration it can fit into the payload space of a commercial rocket. The Ariane 5 rocket family, operated by Arianespace, is the common vehicle choice for heavy satellite launches. The Ariane 5 rocket is capable of transporting up to $20,000 \mathrm{~kg}$ of payload to LEO and its large cargo space is well suited to accommodate the deployable surface mechanism proposed here, and even possibly two devices for two debris removal missions to lower the cost.

The retracted debris collection mechanism and its spacecraft base (chaser) must fit the cargo space of Ariane 5 which is of cylindrical shape, $4.5 \mathrm{~m}$ in diameter and $10 \mathrm{~m}$ in height (Arianespace and Perez (2011)). Based on the information reported by Futron Corporation (2002), we estimate the Ariane 5 launch cost to the target altitude to be on the order of $\$ 10,000 / \mathrm{kg}$. To obtain a realistic weight estimate for the complete system, a working weight for the base satellite must be fixed at this point, thus establishing the weight envelope for the design of the deployable surface and its actuation. The reference spacecraft chosen for our baseline mission is ROGER: it has been proposed for debris 
retrieval from geostationary orbits and has a mass of $620 \mathrm{~kg}$ (Barbee et al. (2012)).

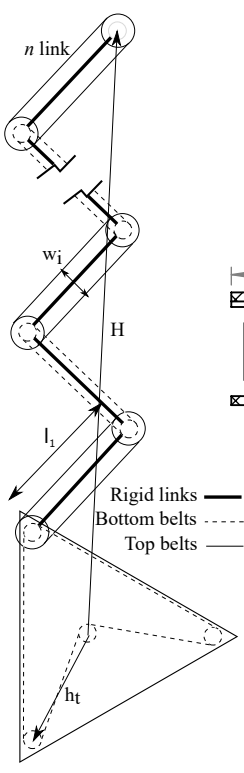

(a)

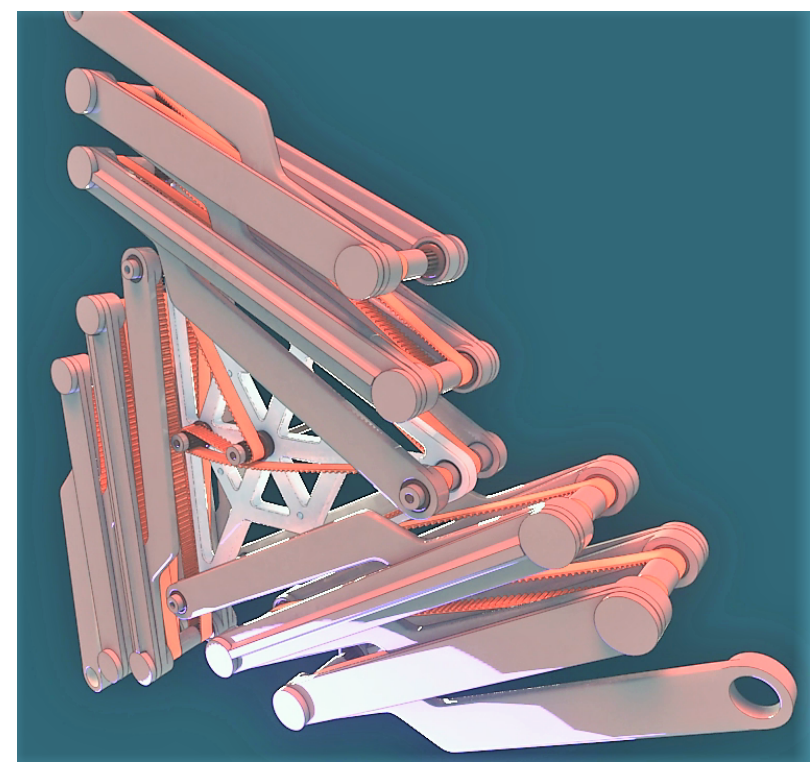

(c)

Figure 3: Belt driven planar triangular deployable mechanism as presented in the work of StOnge and Gosselin (2016b): a) Schematic of a single leg, b) Section of a link with the belts shown as hatched rectangles, c) Artistic view of a face.

Once the device reaches its orbit, the pile of retracted facets will unfold into a small cupola which will then be expanded, using an array of flywheels, to its final large sweeping area. At this point, the ASMDR mission is ready to start; its attitude will be maintained by the flywheels and its attitude, by thruster boosts. In Section 5, we present the results of orbital propagation of the device under the influence of aerodynamic drag and estimate the thruster boost requirements for altitude maintenance over the one-year baseline mission. As elaborated in Section 6, over the course of its mission, the fully deployed device will reorient itself using an array of flywheels. This system will ensure optimal attitude of the cupola to maximize the debris collection area, that is, to maintain its central axis of symmetry and concave surface along and facing the RAM direction, respectively. At the end of the mission, it is expected that the mechanism will retract itself in order to decrease the risk of collision with other satellites while de-orbiting. The de-orbit maneuver would be initiated by the base spacecraft with the fuel reserved for end-of-life. The system is not designed to be recovered, neither the debris that will be captured in it; it will burn up while reentering the atmosphere.

During the operation, we expect collisions of the device with any trackable debris or operational assets to be avoided by relying on active monitoring through the Space Surveillance Network and centralized flight plans coordina- 
tion through the Center for Space Standards and Innovation, analogously to what is proposed in the work of Levin et al. (2012). Probability of these is very low (see Table 2) and the required avoidance maneuvering can be integrated with orbital maintenance necessary for the one-year mission (see Section 5).

\subsection{Mechanical design concept}

Many deployable structures have been previously tested in space (Boccia and Breinbjerg (2012); Pellegrino (2001)), including belt driven mechanisms to deploy solar panels by Li et al. (2013). However, as explained in the work of StOnge and Gosselin (2016b), none can reach a very high expansion ratio while maintaining structural robustness during their deployment. The mechanical concept described in this section uses planar triangular modules assembled in a tessellated curved surface, following the work presented by St-Onge and Gosselin (2016b). The design is first summarized and then adapted to the specific context of ASMDR. To maintain the orientation of the device during a one-year mission, an array of flywheels is introduced and sized.

\subsubsection{Planar triangular deployable modules}

The concept of the planar triangular deployable mechanism driven by belts is illustrated in Fig. 3. A single module of the proposed device is comprised of a rigid triangular base (shown in Figs. 3-a and 3-c), with three legs attached to its vertices, each leg constructed of $n$ links, or leg stages, interconnected via passive revolute joints. Fig. 3-c illustrates the module with the legs nearly fully retracted, each leg made up of 5 links; we observe that the legs can reach a highly compact state over each edge of the inner triangular base. The deployment of the module is achieved as the three legs are extended simultaneously, thanks to a set of belts, drawn in light orange color in Fig. 3-c, transmitting the movement from one leg stage to another. At the end of the deployment, the module maintains its planar "triangular" shape (triangle defined by the three tips of the legs), with the legs stretching out from the vertices of the inner rigid triangle to their fully deployed dimension.

The planar expansion ratio $R_{P}$ of a module is (St-Onge and Gosselin (2016b)):

$$
R_{P}=\frac{H_{D}}{H_{R}}
$$

where $H_{D}$ denotes the deployed radial length of the triangle and $H_{R}$ the retracted one, where the length $H$ is indicated in Fig. 3-a. As was demonstrated by StOnge and Gosselin (2016b) following a number of geometric simplifications, this ratio depends only on the number of links $n$, the width of each link $w$ (assumed all identical, see Fig. 3-b), the triangular base height $h_{t}$ and the length of each link $l_{i}$; it can be optimized as a function of these design variables.

Many triangular modules interconnected through passive revolute joints at each vertex can be assembled in order to create a tessellated surface; planar and curved surface shapes were proposed by St-Onge and Gosselin (2016b). The resulting mechanism has the potential to reach unequaled expansion ratios 
while requiring only one actuator. Moreover, the rigid links and the coordinated unfolding of the legs allow to maintain the triangular shape throughout the deployment process, making it suitable for the deployment of a membrane stretched by this structure. It is this membrane that will provide the primary surface for small debris interception and collection.

\subsubsection{Tessellated cupola unfolding}

To achieve good structural stability, the deployed membrane should not be perfectly planar but rather slightly curved, dome-like. As well, a cupola shape for the device, oriented concave in the RAM direction, is deemed more advantageous for the debris containment in case of rebounds upon impact. To fit the constraints and requirements of our baseline mission scenario, a cupola surface is tessellated with 80 identical triangular modules, shown in their stowed configuration for launch in Fig. 4-b and sequenced in order of assembly in the unfolded cupola in Fig. 4-a. For clarity, this figure shows only the membrane over each module, without the underlying mechanical structure.

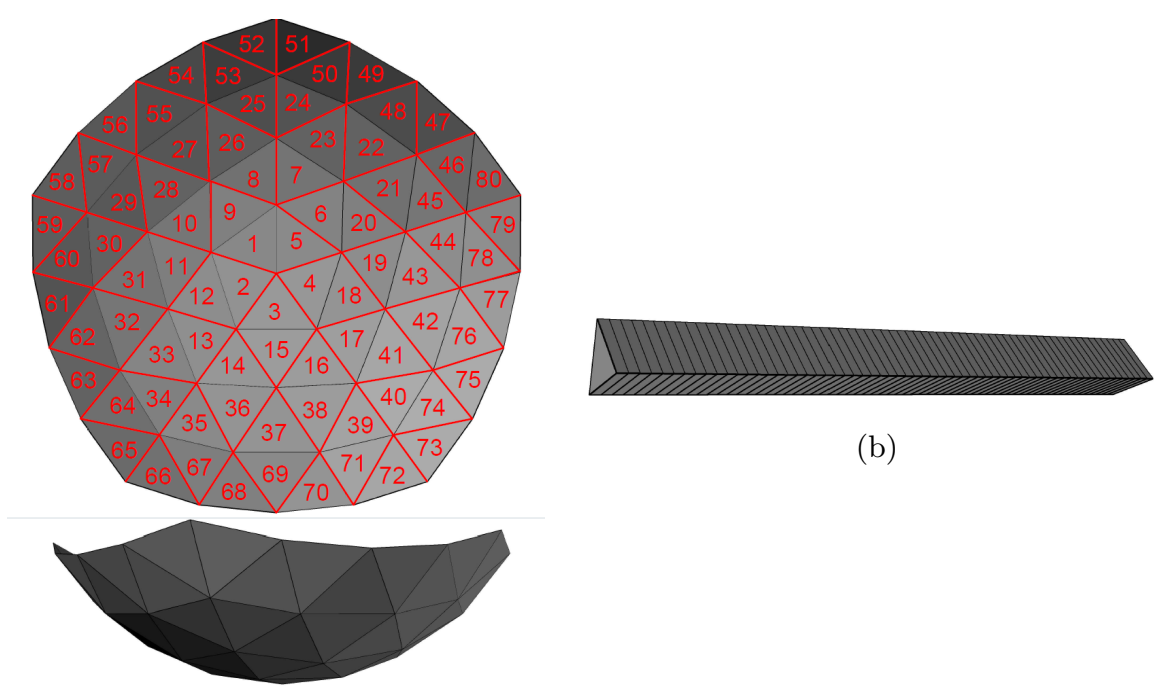

(a)

Figure 4: Cupola schematic model: (a) Unfolding sequence of the triangular modules to form a cupola with a side view of the cupola. (b) Stowed triangular modules for launch and transport.

The unfolding of the triangular modules from their stowed configuration into the assembled cupola in Fig. 4-a is made possible via a custom dual-phased joint design presented by St-Onge and Gosselin (2016b): these joints allow two adjacent modules to unfold by rotating around their shared edge, indicated in red color for each module in Fig. 4-a. The joints are mounted at the leg tips of each module and following the unfolding of a module relative to its neighbor, the corresponding "unfolding" rotation axis is blocked, leaving only the axis normal to the surface of deployment for the relative movement of the modules. The 
detailed design and schematics of the joints are given by St-Onge and Gosselin (2016b). Since the unfolding of the modules occurs once and is not expected to be reversible, the unfolding action is actuated by pre-loaded springs and each vertex' final assembly is assisted by permanent magnets. The second rotation axis of the dual-phased joint is required for the deployment of the surface, together with the aforementioned revolute joints between the leg stages. For this last stage the array of peripheral flywheels (introduced in Section 4.2.3) will serve to extend the multibody structure as described in the work of StOnge and Gosselin (2016a). A cupola of 80 modules would unfold from the stack in Fig. 4-b following the sequential numbering shown in Fig. 4-a. For this kind of non-planar assembly, the motion of the links during deployment must be simulated to ensure that there is no interference with the links of the adjacent modules.

Each triangular module of the deployed cupola will stretch a membrane over it which fills the gaps between the structural legs of the mechanism and, is the primary debris containment surface of the device. The stretched membrane, attaching together all the joints of the module will also stiffen the overall structure and furthermore, reduce the damage risk from an impact of a structural link by holding the links together. It is intended that medium-size debris, if not fully arrested by the membrane, will be sufficiently retarded by it and go through the structure without damaging it. The overall compliance of the device is also advantageous for absorbing part of the impact energy of the debris, as it has been demonstrated for a compliant space manipulator in debris capture (Nishida and Yoshikawa (2003)).

The expansion ratio of the cupola surface, $R_{C}$, is slightly reduced compared to that of the individual triangular modules (Eq. (1)) since it must account for the thickness of each face. As shown by St-Onge and Gosselin (2016b), the effect of the module thickness on the surface expansion ratio decreases with $\frac{t}{a_{R}}$. It can be computed with (St-Onge and Gosselin (2016b)):

$$
R_{C}=\frac{R_{P}+\frac{\sqrt{3}}{\alpha^{2}} \frac{t}{a_{R}}}{1+\frac{\sqrt{3}}{\alpha^{2}} \frac{t}{a_{R}}}
$$

where $\alpha$ is the golden ratio $\left(\frac{1+\sqrt{5}}{2}\right), a_{R}$ is the length of a triangle edge in the retracted configuration and $t$ is the thickness of the modules (see Fig. 3-b). Therefore, the effect of the module thickness on the surface expansion ratio decreases with $\frac{t}{a_{R}}$.

\subsubsection{Attitude Control System}

The deployment of the cupola mechanism is expected to have minimal effect on the spacecraft attitude since this action is radially symmetrical. However, the unfolding of each module into the assembled cupola will generate non-negligible attitude perturbations. An Attitude Control System (ACS) of the satellite base can be used to compensate for these perturbations during the initial set-up phase of the mission. Depending on the design of ACS, the same system may 
be suitable for attitude maintenance of the fully deployed device during the one-year mission. However, here we propose a distributed attitude maintenance system to compensate for the angular momentum transfer from the impacting debris, namely an array of small flywheels mounted directly on the cupola, based on the concept presented in the work of St-Onge and Gosselin (2016a). This approach was proven effective for control of both the orientation and the deployment of a free-floating multi-body mechanism. Its redundancy also allows an approximate control of the device even in the case of potential damage as a result of debris impact.

The inertial properties of the flywheels as well as the driving motor characteristics can be optimized to ensure that sufficient torque and angular momentum are available to maintain a proper average orientation of the cupola device throughout its mission. Since the angular momentum imparted by the debris is expected to be primarily about axes in the plane perpendicular to the RAM direction, and with the view to maintaining the axial symmetry of the device, we suggest an optimized arrangement of the flywheels on the cupola along its perimeter, as illustrated in Fig. 5. The flywheels are mounted on the faces of the peripheral triangular modules, with their axes normal to the modules, thus resulting in the maximum possible angular momentum generated about the radial $e_{R}$ and normal $e_{N}$ directions of the orbit. The net angular momentum, $\mathbf{H}_{f}$, produced by the action of the flywheels can be computed with:

$$
\mathbf{H}_{f}=\sum_{i=1}^{n_{f}} C_{f i} \mathbf{e}_{i}
$$

where $n_{f}$ is the number of flywheels, $C_{f i}$ is the angular momentum of the $i$ th flywheel and $\mathbf{e}_{i}$ is the unit vector in the direction of the axis of the $i$ th flywheel. In the following section, an off-the-shelf flywheel model is suggested for the baseline mission scenario and the maximum angular momentum of the array in the $e_{R^{-}} e_{N}$ plane computed.

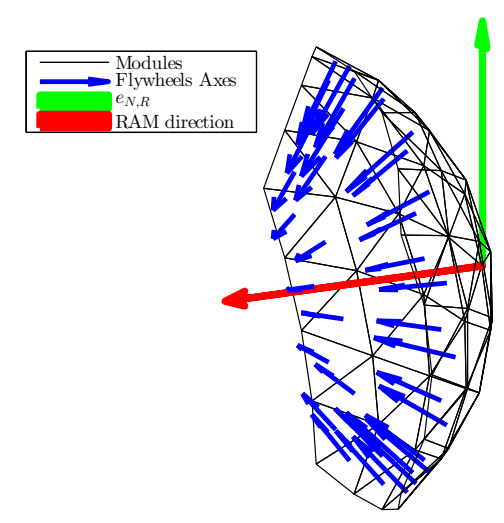

Figure 5: Assembled cupola with flywheel rotation axes (in blue), RAM direction (in red) and perpendicular direction (in green) 


\subsection{Design considerations for ASMDR}

The following main design choices need to be considered for the deployable cupola device: geometry, material and number of the structural links, the choice of belts, the material for the collecting membrane and the size/model of flywheels for attitude maintenance.

Starting with the structural links, two geometric parameters must be fixed before optimizing the triangular module design for this ASMDR application: the width of the links $w$ and their thickness $t$ (see Fig. 3-b). Since the links are not designed to resist a hyper-velocity impact with a debris, the load that they must withstand is the tension in the transmission belts used for the leg deployment. The belt length is expected to be in the range of 1 to $9 \mathrm{~m}$ (twice the estimated length of the mechanism's first link) and the belt material must be robust to the harsh environment of space. A product well suited for the present application is the carbon reinforced timing belt made by Gates (Gates (2009)). The main advantage of a carbon reinforced matrix is that it is not sensitive to temperature variations. As an example, for a Gates belt of $21 \mathrm{~mm}$ width and $4.2 \mathrm{~m}$ length, the required tension is $249 \mathrm{~N}$ to transmit a maximum power of 75 Watts; this belt has a linear mass density of $150 \mathrm{~g} / \mathrm{m}$. The mechanism links must then be sized to withstand a total tension load of approximately $500 \mathrm{~N}$.

As discussed in Section 4.1, the main constraint on the size of the device is due to the available payload space of the launcher, which for the stowed configuration of our device is the diameter of the Ariane 5 cargo bay $(4.5 \mathrm{~m})$. The radial length of the mechanism $H_{R}$ is then limited to half of this value. Since the final geometric design of the mechanism will likely take advantage of long links, their width must be large enough to withstand the tension in the belts; it is chosen to be $60 \mathrm{~mm}$ here. In the end, only the number of links of each leg $n$ and the length of the first link $l_{1}$ remain as design variables for optimizing the expansion ratio $R_{P}$. Kinematic analysis and simulations were conducted in order to validate the feasibility of the optimized parameters.

With the link length output from the optimization process, structural analysis of the triangular module could be carried out. Long links $\left(l_{1}>2.5 \mathrm{~m}\right)$ turn out to be too weak to resist the belt tension and using more than 30 links is deemed risky, due to the numerous joints and probability of failure. Therefore, the selected configuration has 30 links per leg with the first one measuring 2.1 m. The planar expansion ratio of this mechanism calculated using Eq. (1) is $R_{P}=23$. With two $21 \mathrm{~mm}$ width timing belts, one on top of the other for each link, a minimal thickness $t=75 \mathrm{~mm}$ is required for the whole stage (see Fig. 3b). As noted earlier, the launcher cargo bay is $10 \mathrm{~m}$ high and the satellite base (ROGER) takes approximately $2 \mathrm{~m}$, leaving room for more than one hundred planar modules in a stacked arrangement, as shown in Fig. 4-b. In order to fit the payload weight budget of Ariane 5, allowing for transport of two devices, a cupola design composed of 80 modules is proposed. This configuration takes up $8 \mathrm{~m}$ in height (including ROGER) and yields an unfolded cupola surface of $527 \mathrm{~m}^{2}$ and a fully deployed cupola surface of $0.27 \mathrm{~km}^{2}$. The final expansion ratio obtainable with the device is only slightly smaller than the ideal $R_{P}$, which is estimated to be $R_{C}=22.7$, using Eq. (2) (with $a_{R}=3.9 \mathrm{~m}$ ). 
As shown in Fig. 3-b, the links are constructed with two sections, one on each side of the belts. This geometry increases the robustness of the mechanism in the plane of the triangular modules, helping both to resist belt tension and, for modules near the periphery of the cupola, to withstand at least some of the debris impacts. We estimate the critical (buckling) load for the links by assuming that their longitudinal stiffness matches that of isotropic aluminum alloy. With the geometric properties for the section of Fig. 3-b and the dimensions cited earlier, we estimate the critical load that the link can withstand at approximately $70 \mathrm{kN}$, much higher than what is required to support the tension in the belts. The mass of such a link, made of carbon fiber composite is about $0.65 \mathrm{~kg}$. Including the estimated mass $(2 \mathrm{~kg})$ of the inner triangle of the deployable module, the total mass of the triangular module structure is approximately $60 \mathrm{~kg}$. Using the belt selection made earlier to transmit the unfolding motion to the tip of each of the three legs adds $14 \mathrm{~kg}$ to the mass of each module.

As noted previously, the structural elements of the device are not intended to resist hyper-velocity impacts, and fortunately, the probability of a debris hitting any one of the structural links is low. For a single deployed triangular module, the total surface covered by the membrane on top of the structure is $3430 \mathrm{~m}^{2}$ while the structure itself covers only $6.59 \mathrm{~m}^{2}$. Therefore, there is a less than $0.2 \%$ chance of the debris impacting on the structure. The membrane material, however, must be selected to sustain and contain the debris impacts, without creating new debris. Relevant information on the available materials and configurations for such a membrane can be found in the review published by Christiansen (2003) for NASA. We propose to use layers of fabric material

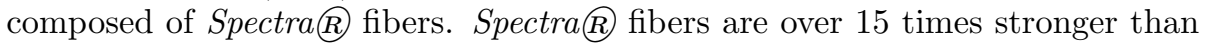
steel while as durable as polyester, lighter than carbon and have a $40 \%$ higher specific resistance than Aramid@ (Honeywell (2013)). These fiber-reinforced plastics were extensively tested with hypervelocity projectiles, demonstrating that even with strong damage $(80 \%)$ the membrane will delaminate and rip, rather than exploding and creating new small debris (Riedel et al. (2006)). Even in the event of ripping the membrane material, the impact dissipates a lot of energy and slows down the larger debris (Christiansen (2003)). The density of a Spectra $1000 \mathrm{film}$ is $970 \mathrm{~kg} / \mathrm{m}^{3}$ and, with the remaining mass in the payload budget of $35 \mathrm{~kg}$ per module, allows for the maximum membrane thickness of $10.5 \mu \mathrm{m}$. The final weight budget of the device is presented in Tab. 1 .

The last of the mechanical system components to be specified for the baseline mission are the flywheels mounted on the cupola, as proposed in Section 4.2.3. The Rockwell Collins ${ }^{\mathrm{TM}}$ MWI 100-100/100 ${ }^{2}$ model was selected for the high angular momentum that it can deliver $\left(100 \mathrm{~kg}-\mathrm{m}^{2}-\mathrm{s}^{-1}\right)$, within a reasonable weight envelope $(16 \mathrm{~kg})$. As described earlier, one flywheel is to be mounted on each module of the cupola along its perimeter - faces 46 to 80 in Fig. 4-a - for a total of 35 flywheels. This configuration is optimal for maximizing the angular

\footnotetext{
2 https://www.rockwellcollins.com/Products_and_Services/Defense/Platforms/ Space/Magnetic_Bearing_Momentum_and_Reaction_Wheel.aspx
} 
Table 1: Mass budget for the deployable device.

\begin{tabular}{r|c}
\hline Single module mass & $74 \mathrm{~kg}$ \\
Single module membrane mass & $35 \mathrm{~kg}$ \\
Number of faces & 80 \\
Total mass of the flywheels & $560 \mathrm{~kg}$ \\
Base satellite mass & $620 \mathrm{~kg}$ \\
Total mass & $\mathbf{9 9 0 0 ~ k g}$ \\
Surface & $0.27 \mathrm{~km}^{2}$ \\
Projected disk diameter & $400 \mathrm{~m}$ \\
\hline
\end{tabular}

momentum storage in the flywheels in the radial-normal directions needed to compensate for the expected angular momentum transfer from the impacting debris. The angular momentum of the flywheels array about an arbitrary axis normal to the direction of motion, i.e., in the radial-normal plane, is computed using:

$$
H_{f, R N}=\sum_{i=1}^{35} C_{f i} \mathbf{e}_{i} \cdot \mathbf{e}_{R N}
$$

For the model of flywheels selected, the corresponding maximum value of $H_{f, R N}$ evaluates to $2202 \mathrm{~kg}-\mathrm{m}^{2}-\mathrm{s}^{-1}$.

\subsection{Expected performance}

Using MASTER-2009 software for the frontal area of the device of approximately $0.27 \mathrm{~km}^{2}$ (projected disc of radius $200 \mathrm{~m}$ ), orbiting at $819 \mathrm{~km}$ altitude, we estimate that the proposed debris collection device would cross on the order of $1.3 \times 10^{9}$ objects along its path, during a one-year mission (see Tab. 2). Excluding meteoroids, which account for approximately $22 \%$ of the impacts, about $1.0 \times 10^{9}$ pieces of debris would be recovered, or $1.2 \%$ of all small-to-medium sized objects predicted to exist in this orbit. Among these, an estimated 112 objects are expected to be in the critical size range $0.5-5 \mathrm{~cm}$. With a total mass of 9,900 kg budgeted for the device, as per Tab. 1, and a previously cited launch cost estimate of $\$ 10,000 / \mathrm{kg}$, the resulting cost of small debris removal with the device is $\$ 0.1 /$ debris, but it is an enormous $\$ 880,000$ million/debris for critical size objects.

Note that the fuel weight and cost required for orbit maintenance of the cupola, addressed in the following section, have not been included in the present budget estimates, nor the cost of the spacecraft system itself. However, the $\Delta v$ budget for orbital maintenance is computed, and we suggest a thruster technology to achieve it.

\section{Orbit Maintenance of ASMDR System}

The proposed device will have a high frontal area in order to maximize the debris collection performance, while satisfying the constraints of the launcher chosen. In this section, we present the results of an orbital propagation analysis to estimate the expected acceleration due to drag at the mission altitude and the $\Delta v$ needed to maintain the orbit. 
The orbital equation of motion of a satellite, in an Earth-centered inertial (ECI) frame, is:

$$
\ddot{\mathbf{r}}(t)=-\frac{\mu}{r(t)^{3}} \mathbf{r}(t)+\sum_{i} \mathbf{a}_{i}(t, \mathbf{r}(t))
$$

where $\mathbf{r}$ is the position of the satellite as a function of time $t, r=\|\mathbf{r}\|, \mu$ is the Earth's gravitational parameter, $\mathbf{a}_{i}$ represents the non-gravitational accelerations, and assuming a perfectly uniform spherical Earth, ignoring Earth's oblateness and third-body interactions.

At LEO altitudes, aerodynamic drag constitutes the primary environmental effect responsible for orbital decay. The acceleration due to aerodynamic drag is:

$$
\mathbf{a}_{\mathrm{drag}}=-\frac{1}{2} \frac{C_{d} A}{m} \rho v_{\mathrm{rel}}^{2} \frac{\mathbf{v}_{\mathrm{rel}}}{\left\|\mathbf{v}_{\text {rel }}\right\|}
$$

where $C_{d}$ is the drag coefficient of the satellite, $A$ is the satellite cross-sectional area normal to the incident atmospheric flow, $m$ is the spacecraft mass, $\rho$ is the atmospheric density, and $\mathbf{v}_{\text {rel }}$ is the relative velocity of the satellite with respect to the atmosphere, assumed here to be co-rotating with Earth.

In our analysis, atmospheric densities were obtained using the DTM-2013 model (Bruinsma (2015)), a three-dimensional model of the thermosphere. DTM2013 uses the F30 solar flux as a proxy for solar activity along with the $K_{p}$ geomagnetic index (Bruinsma (2015)). The drag coefficient is a parameter related to the way the gas particles hit and slide on the satellite surface, and was assumed to be 2.2 here. As per design definition of Section 4, the cross-sectional area $A$ was set to a constant value of $\pi 200^{2} \mathrm{~m}^{2}$.

Atmospheric density is largely dependent on solar activity, which can vary drastically during the course of an 11-year solar cycle. Therefore, to predict the acceleration due to drag experienced by the cupola, the solar activity of 2009 was chosen to represent the planned mission year of 2020. However, as 2009 was a year of remarkably low activity (NASA (2009)), a second propagation was carried out using data for 2000, a year of high solar activity, emulating a mission year of 2022 .

Fig. 6 shows the expected drag acceleration that the cupola would experience after propagating the orbit of the cupola for one year, though countering the effect of drag with a continuous thrust so that no orbital decay occurs. The actual F30 and $K_{p}$ indices corresponding to years 2009 and 2000 were used as inputs to DTM-2013. Fig. 6 shows that the expected drag acceleration is of the order of $10^{-6} \mathrm{~m} / \mathrm{s}^{2}$ for 2009 (grey line), while it can vary from $10^{-6}$ to $10^{-4} \mathrm{~m} / \mathrm{s}^{2}$ for 2000 (black line). The differences and cyclical variations seen in the two simulations are due to solar activity. For the year 2000, the variations in the solar flux are strong, leading to large fluctuations in atmospheric density. In 2009, solar activity is low and the solar flux varies less, and seasonal cycles therefore dominate. Integrating the acceleration values over the entire year, an estimate of the $\Delta v$ budget was obtained: 48 and $513 \mathrm{~m} / \mathrm{s}$ for 2009 and 2000, respectively. An ion propulsion continuous thrust system can be feasible for a mission in either scenario and we expect the additional weight of fuel to be less 
than $4 \%$ of the dry weight for the higher $\Delta v$ requirement. With the historic knowledge and future predictions of the solar cycles, however, the mission can be planned for a period of low solar activity in order to minimize the amount of fuel needed to maintain the desired orbit.

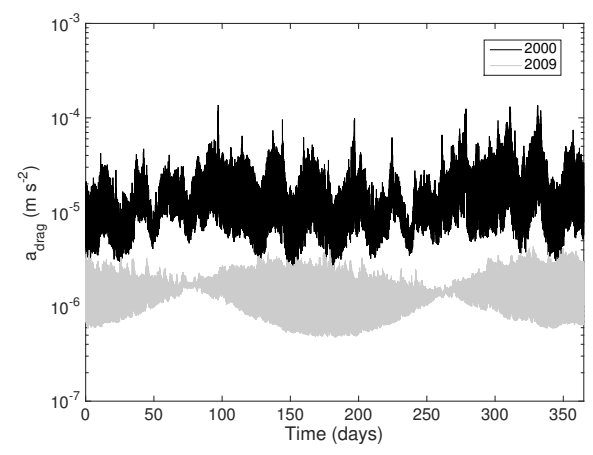

Figure 6: Atmospheric drag acceleration for a $400 \mathrm{~m}$ diameter cupola in $819 \mathrm{~km}$ orbit, one-year mission.

\section{Attitude Maintenance of ASMDR System}

As alluded to earlier, the intended orientation of the device is such that its central axis is aligned with the ram direction, ensuring maximum membrane exposure to the incoming debris, the directional flux of which peaks at zero azimuth and elevation angles, as seen from MASTER-2009. Due to the large size of the cupola, however, the device may experience significant angular momentum transfer from the impacting debris, which if uncompensated, will disturb the cupola's orientation.

To estimate this effect, the framework for stochastic modeling of hypervelocity impacts proposed in the work of Sagnières and Sharf (2017) was employed to propagate the cupola's attitude under bombardment of debris and meteoroids. The model combines MASTER-2009 impact fluxes as a function of impactor mass, velocity and direction for the desired input orbit to produce probability density functions and generate random collisions during the propagation time frame. It sets up the transfer of the impactor momentum to the system (the cupola in our case) as a stochastic process called a compound Poisson process in the stochastic differential equation for attitude motion. A Monte Carlo simulation is then performed in order to obtain probability distributions for the rotational parameters and their evolution with time.

In the envisioned mission scenario, the trackable debris, larger than $5 \mathrm{~cm}$, will be avoided; however, collisions with smaller impactors, down to $1 \mu \mathrm{m}$, are numerous, estimated to approximately 1.3 billion during a one-year mission. The expected number of collisions from relevant categories are presented in Tab. 2, using the mean debris size to mass conversion from the work of Vance and Mense (2013). Because of the combined effects of the symmetry of the cupola and of the directional flux of the incoming debris, and the very high 
Table 2: Object encounter probability over a one-year mission obtained from MASTER-2009.

\begin{tabular}{r|r|c|c}
\hline Size $(\mathrm{cm})$ & Mass $(\mathrm{g})$ & Probability $(\%)$ & Objects \# \\
\hline$<0.5$ & $<0.05$ & 100 & $1.3 \times 10^{9}$ \\
0.5 to 5 & 0.05 to 30 & $8.7 \times 10^{-6}$ & 112 \\
$>5$ & $>30$ & $3.2 \times 10^{-7}$ & 4 \\
\hline
\end{tabular}

number of collisions, we expect the impacts with debris smaller than $1 \mathrm{~g}$ to average out to an almost null disturbance in the long-term. The Monte Carlo simulations carried out, using the MASTER-2009 fluxes for the year 2020 and considering only collisions with impactors having a mass less than $1 \mathrm{~g}$, confirm this. Figure 7 shows a histogram of the final rotational kinetic energy of the cupola after one year for 241 completed simulations. The initial rotation of the device was set to $360^{\circ}$ per orbital period so as to keep the orientation constant in the orbital frame. The deviation from the initial kinetic energy is within $10 \%$ in every case and within $5 \%$ in $97 \%$ of cases. This shows that impacts from very small debris will not have a strong influence on the orientation and rotation of the cupola, and that the flywheels will easily be able to compensate for the slight deviations.

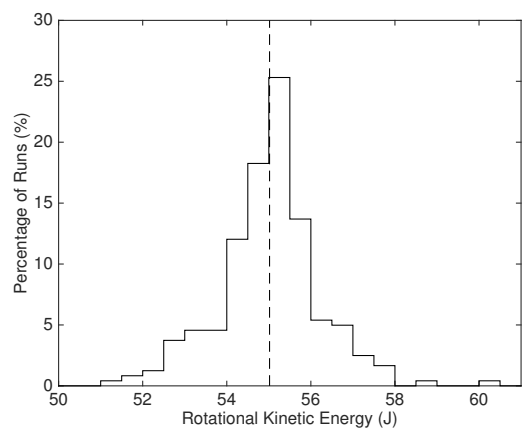

Figure 7: Final rotational kinetic energy histogram for a one-year propagation of collisions with debris $<1 \mathrm{~g}$.

The most relevant category of debris significantly disturbing the orientation of the device is the $0.5-5 \mathrm{~cm}$ size range. As already noted, approximately 112 collisions from this critical range are expected to occur throughout the mission, as seen from Tab. 2, and these collisions will be the most problematic as their effect will not average out. Fig. 8 shows the probability, derived from the MASTER-2009 fluxes, of the angular momentum transfer for one such collision with an impactor of mass between 0.05 and $30 \mathrm{~g}$, the mean and most probable angular momentum transfer being approximately 500 and $100 \mathrm{~kg}-\mathrm{m}^{2}-\mathrm{s}^{-1}$, respectively. The maximum angular momentum transfer experienced is approximately $10,000 \mathrm{~kg}-\mathrm{m}^{2}-\mathrm{s}^{-1}$.

In Section 4.3, we had estimated the maximum angular momentum that can be compensated in the radial-normal plane with the distributed flywheels arrangement and a particular flywheel system to be $2202 \mathrm{~kg}-\mathrm{m}^{2}-\mathrm{s}^{-1}$, or four times the mean angular momentum transfer from a medium-size debris impact. These 


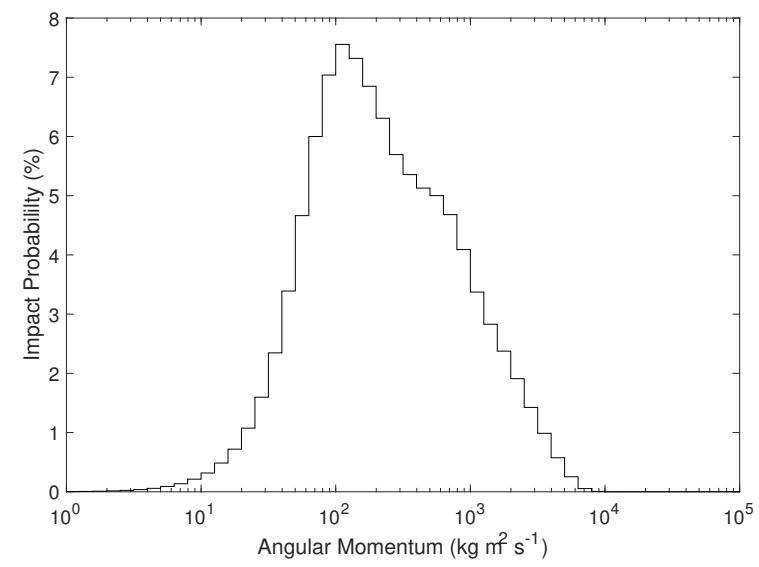

Figure 8: Angular momentum transferred from debris collisions.

results thus confirm the feasibility of compensating for attitude perturbations resulting from debris impacts expected during the proposed mission.

\section{Conclusion}

It was argued in this paper that small-to-medium size untrackable debris pose a serious threat to many operational spacecraft, especially considering their numerousness in LEO. A review of existing solutions for active debris removal revealed that there are no feasible means at present to deal with this problem. This paper put forward one possible solution based on a novel deployable mechanism and actuation method for the removal of small-to-medium size debris. It is also worth noting and although not explored in this work, the proposed cupola concept could also be useful as small debris detection device, along the lines of dust detector concepts that have been proposed and used in space in the past Bauer et al. (2014); Kobayashi et al. (2011).

Following realistic assumptions on the mission parameters, the study shows that a cupola comprised of 80 triangular faces, each deployed with a belt-driven planar mechanism with an expansion ratio of 23 can provide a collection area of $0.27 \mathrm{~km}^{2}$ to capture small debris. The mission planning and the design of the device also address the problems of orbit and attitude maintenance over a nominal one-year mission, the latter achieved with an array of 35 flywheels, mounted on the periphery of the cupola. The estimated orbit maintenance requirements are $\Delta v$ of $48 \mathrm{~m} / \mathrm{s}$ and $513 \mathrm{~m} / \mathrm{s}$, for low and high solar activity mission years, to maintain the cupola in the $819 \mathrm{~km}$ orbit.

The technical analysis detailed in this paper demonstrates that the proposed solution and mission plan are feasible and can be accommodated within the existing launch capabilities. We predict that the system will retrieve at least $1.0 \times 10^{9}$ small to medium size objects during its one-year mission. At the same time, as with any space mission, the proposed ASMDR solution comes with a 
number of associated risks, such as, mechanism deployment failure, structural failure during operation, attitude stabilization failure, and in the worst-case scenario, the risk of adding $10,000 \mathrm{~kg}$ of mass to the orbital debris environment. Moreover, juxtaposing the $\$ 880,000 /$ debris cost estimate of removing debris in the $0.5-5 \mathrm{~cm}$ size range against the value analysis result reported by Vance and Mense (2013) of $\$ 14,500 /$ debris value for removing such a debris leads one to conclude that the proposed mission concept is not economically viable at this time. Nevertheless, the present study paves the way for future mission concepts for removal of small-to-medium-sized debris to ensure continued safety in space.

\section{Acknowledgment}

The authors would like to acknowledge the financial support of the Natural Sciences and Engineering Research Council of Canada (NSERC), Hydro-Quebec and the Canada Research Chair Program. The artistic view of Fig. 3-c is a courtesy of Jean-Philippe Toutan.

\section{References}

Aghili, F., 2012. Active Orbital Debris Removal Using Space Robotics. International Symposium on Artificial Intelligence, Robotics and Automation in Space (i-SAIRAS) , 1-9.

Anz-Meador, P., Shoots, D., 2016. Recent NOAA-16 Satellite Breakup. Orbital Debris Quarterly News 20.

Arianespace, Perez, E., 2011. Ariane 5 User's Manual. Arianespace SA. , 271.

Barbee, B.W., Alfano, S., Piñon, E., Gold, K., Gaylor, D., 2012. Design of Spacecraft Missions to Remove Multiple Orbital Debris Objects, in: 35th Annual AAS Guidance and Control Conference, Breckenridge. pp. 1-19.

Bauer, W., Romberg, O., Wiedemann, C., Drolshagen, G., Vörsmann, P., 2014. Development of in-situ Space Debris Detector. Advances in Space Research 54, 1858-1869.

Biesbroek, R., S.T.H.S.J.W.K., Innocenti, L., 2013. Deorbit CDF Study: A Design Study for the Safe Removal of a Large Space Debris, in: 64th International Astronautical Congress, Beijing, China.

Boccia, L., Breinbjerg, O., 2012. Antenna Basics, in: Space Antenna Handbook. John Wiley \& Sons, Ltd, pp. 1-35.

Bonnal, C., Ruault, J.m.M., Desjean, M.c.C., 2013. Active debris removal: Recent progress and current trends. Acta Astronautica 85, 51-60.

Botta, E., Sharf, I., Misra, A., 2016. Evaluation of Net Capture of Space Debris in Multiple Mission Scenarios, in: 26th AAS/AIAA Space Flight Mechanics Meeting, Nappa, CA. 
Bruinsma, S., 2015. The DTM-2013 thermosphere model. Journal of Space Weather and Space Climate 5, A1.

Christiansen, E., 2003. Meteoroid/Debris Shielding. Technical Report August. NASA. Houston.

Christiansen, E.L., Hyde, J.L., Bernhard, R.P., 2004. Space Shuttle debris and meteoroid impacts. Advances in Space Research 34, 1097-1103.

Dudziak, R., Tuttle, S., Barraclough, S., 2015. Harpoon technology development for the active removal of space debris. Advances in Space Research 56, $509-$ 527. Advances in Asteroid and Space Debris Science and Technology - Part 1.

Dunn, M.J., 2014. Space Debris Removal. US Patent 8800933 B2.

ESA, . Hypervelocity impacts and protecting spacecraft.

Flegel, S.K., Gelhaus, J., Möckel, M., Wiedemann, C., Krag, H., Klinkrad, H., Vörsmann, P., 2011. Multi-layer insulation model for MASTER-2009. Acta Astronautica 69, 911-922.

Futron Corporation, 2002. Space Transportation Costs: Trends in Price Per Pound to Orbit 1990-2000. Technical Report. Futron Corporation.

Ganguli, G., Crabtree, C., Rudakov, L., Chappie, S., 2011. A Concept For Elimination Of Small Orbital Debris. Nature 473, 221-225.

Gates, 2009. Polychain GT Carbon Belt Drive Design Manual. Technical Report. Gates Corporation. Denver.

Hirzinger, G., Landzettel, K., Brunner, B., Fischer, M., Preusche, C., Reintsema, D., Albu-Schäffer, A., Schreiber, G., Steinmetz, B.M., 2004. DLR's robotics technologies for on-orbit servicing. Advanced Robotics 18, 139-174.

Honeywell, 2013. Honeywell Spectra @ Fiber Capability Guide. Technical Report. Honeywell.

Kessler, D.J., Cour-Palais, B.G., 1978. Collision frequency of artificial satellites: The creation of a debris belt. Journal of Geophysical Research 83, 2637-2646.

Klinkrad, H., Johnson, N., 2009. Space debris environment remediation concepts, in: International Conference on Orbital Debris Removal, Chantilly, VA.

Kobayashi, M., Miyachi, T., Nakamura, M.H., 2011. Cosmic Dust Detector Capable of Measuring Hypervelocity Speed Using Piezoelectric PZT, in: 42nd Lunar and Planetary Science Conference, The Woodlands. 
Krag, H., Klinkrad, H., Oswald, M., Stabroth, S., Wiedemann, C., 2007. Analysing the risk increase in LEO due to recent major fragmentation events. European Space Agency, (Special Publication) ESA SP .

Lappas, V., Adeli, N., Visagie, L., Fernandez, J., Theodorou, T., Steyn, W., Perren, M., 2011. CubeSail: A low cost CubeSat based solar sail demonstration mission. Advances in Space Research 48, 1890-1901.

Levin, E., Pearson, J., Carroll, J., 2012. Wholesale debris removal from LEO. Acta Astronautica 73, 100-108.

Li, J., Yan, S., Guo, F., Guo, P., 2013. Effects of damping, friction, gravity, and flexibility on the dynamic performance of a deployable mechanism with clearance. Proceedings of the Institution of Mechanical Engineers Part CJournal of Mechanical Engineering Science 227, 1791-1803.

Liou, J.C., 2011a. A Note on Active Debris Removal. Orbital Debris Quarterly News 15.

Liou, J.C., 2011b. An active debris removal parametric study for LEO environment remediation. Advances in Space Research 47, 1865-1876.

Mandeville, J.C., Rival, M., Alby, F., 1999. Secondary impact generated particles: implications for the orbital debris population. Advances in Space Research 23, 89-94.

Mason, J., Stupl, J., Marshall, W., Levit, C., 2011. Orbital debris-debris collision avoidance. Advances in Space Research 48, 1643-1655.

Nakamura, M., Kitazawa, Y., Matsumoto, H., Okudaira, O., Hanada, T., Sakurai, A., Funakoshi, K., Yasaka, T., Hasegawa, S., Kobayashi, M., 2015. Development of in-situ micro-debris measurement system. Advances in Space Research 56, 436-448.

NASA, 2009. Deep solar minimum. Last Checked 2016-11-29.

Nguyen-Huynh, T.C., Sharf, I., 2013. Adaptive Reactionless Motion and Parameter Identification in Postcapture of Space Debris. Journal of Guidance, Control, and Dynamics 36, 404-414.

Nishida, S., Yoshikawa, T., 2003. Space debris capture by a joint compliance controlled robot, in: IEEE/ASME International Conference on Advanced Intelligent Mechatronics, Kobe. pp. 496-502.

Nock, K.T., McRonald, A.D., Maynard Aaron, Maynard, K., 2003. Balloon device for lowering space object orbits. US Patent 6830222B1.

Pellegrino, S., 2001. Deployable Structures. Springer Vienna, Vienna. 
Riedel, W., Nahme, H., White, D.M., Clegg, R.A., 2006. Hypervelocity impact damage prediction in composites : Part II experimental investigations and simulations. International Journal of Impact Engineering 33, 670-680.

Ryan, S., Christiansen, E.L., 2011. A ballistic limit analysis programme for shielding against micrometeoroids and orbital debris. Acta Astronautica 69, $245-257$.

Sagnières, L., Sharf, I., 2017. Stochastic Modeling of Hypervelocity Impacts in Attitude Propagation of Space Debris. Advances in Space Research .

Schmitz, M., Fasoulas, S., Utzmann, J., 2015. Performance model for spacebased laser debris sweepers. Acta Astronautica 115, 376-383.

Shan, M., Guo, J., Gill, E., 2016. Review and comparison of active space debris capturing and removal methods. Progress in Aerospace Sciences 80, 18 - 32 .

St-Onge, D., Gosselin, C., 2016a. Dynamic modelling of a four-bar free floating mechanism with passive joints and flywheel actuators, in: International Symposium on multi-body system dynamics, Montreal. pp. 1-10.

St-Onge, D., Gosselin, C., 2016b. Synthesis and Design of a One Degreeof-Freedom Planar Deployable Mechanism With a Large Expansion Ratio. ASME Journal of Mechanisms and Robotics 8, 021025-021025-9.

Taylor, R., Gravseth, I., Turse, D., Keller, P., Hulse, M., Richardson, D., 2013. Method for removing orbital objects from orbit using a capture net for momentum transfer.

Ting Wang, 2010. Analysis of Debris from the Collision of the Cosmos 2251 and the Iridium 33 Satellites. Science and Global Security 18, 87-118.

Vance, L., Mense, E., 2013. Value analysis for orbital debris removal. Advances in Space Research 52, 685-695.

Williamsen, J.E., Schonberg, W.P., Evans, H., Evans, S., 2008. A comparison of NASA, DoD, and hydrocode ballistic limit predictions for spherical and non-spherical shapes versus dual- and single-wall targets, and their effects on orbital debris penetration risk. International Journal of Impact Engineering $35,1870-1877$.

Zhai, G., Zhang, J.R., 2012. Space tether net system for debris capture and removal, in: 4th International Conference on Intelligent Human-Machine Systems and Cybernetics, Nanchang. pp. 257-261. 